\title{
Enzymatic synthesis of Medium- and Long-Chain Triacylglycerols (MLCT): Optimization of process parameters using response surface methodology.
}

\begin{abstract}
The main objective of this study was to understand the effects and relationship amongst four factors, which are reaction temperature, reaction time, enzyme load, and substrate mole ratio with the purpose of producing healthy functional cooking oil for long-term dietary treatment. Lipozyme RM IM lipase-catalyzed esterification of medium- and long-chain triacylglycerols (MLCT) from glycerol and mixtures of capric and oleic acid was optimized using response surface methodology (RSM) with a five-level, four-factorial design. Reaction temperature, reaction time, and substrate mole ratio strongly affected MLCT synthesis $(\mathrm{P}<0.01)$. However, enzyme load did not have a significant $(\mathrm{P}>0.01)$ effect on MLCT yield. Comparison between predicted and experimental value from central composite rotatable design optimization procedures revealed good correlation, implying that the reduced cubic polynomial model with backward elimination statistically expressed the percent MLCT yield obtained. The optimum MLCT yield was $59.76 \%$ by using $10 \mathrm{wt} \%$ enzyme load, reaction temperature of $70^{\circ} \mathrm{C}$, reaction time of $14 \mathrm{~h}$, and substrate mole ratio of 3.5:1. Experiments to confirm the predicted results using the optimal parameters showed an MLCT yield of $56.35 \%$ $(n=2)$. The choice on the types of fatty acids used in MLCT optimization work greatly influenced the physical and chemical properties of MLCT oil produced. The refined MLCT oil characteristics study showed this oil is suitable to be used for cooking/frying purposes as a high-value added product.
\end{abstract}

Keyword: Capric acid; Medium- and long-chain triacylglycerols (MLCT); Oleic acid; Response surface methodology (RSM). 